\title{
Propagation of Bose-Einstein condensates in a magnetic waveguide
}

\author{
A.E. Leanhardt, A.P. Chikkatur, D. Kielpinski, Y. Shin, T.L. Gustavson, W. Ketterle, D.E. Pritchard \\ Department of Physics, MIT-Harvard Center for Ultracold Atoms, and Research Laboratory of Electronics, \\ Massachusetts Institute of Technology, Cambridge, Massachusetts, 02139
}

(Dated: October 26, 2018)

\begin{abstract}
Gaseous Bose-Einstein condensates of $2-3 \times 10^{6}$ atoms were loaded into a microfabricated magnetic trap using optical tweezers. Subsequently, the condensates were released into a magnetic waveguide and propagated $12 \mathrm{~mm}$. Single-mode propagation was observed along homogeneous segments of the waveguide. Inhomogeneities in the guiding potential arose from geometric deformations of the microfabricated wires and caused strong transverse excitations. Such deformations may restrict the waveguide physics that can be explored with propagating condensates.
\end{abstract}

PACS numbers: 03.75.Fi, 03.75.Be, 39.20.+q

Progress in the field of atom optics depends on developing improved sources of matter waves and advances in their coherent manipulation. Extended samples of coherent atoms were introduced to the field in 1995 with the realization of Bose-Einstein condensation in dilute alkali vapors [1]. Early success in coherently controlling condensates was achieved by applying optical and radio frequency fields [1]. Further efforts to increase the repertoire of coherent manipulation techniques for ultracold atomic vapors pursued miniaturizing the current carrying structures often used in their study [2, 3. Following the successful trapping and guiding of thermal atoms using self-supported miniature wires [4, 5, 6] and substratesupported microfabricated wire arrays [7, 8, 9, 10], recent experiments merged wiretraps on the millimeter scale [11] and microfabricated electronic devices 12, 13] with Bose-Einstein condensation. This has opened up a front on which further techniques for coherent condensate transport and manipulation can be explored. While previous demonstrations of condensate guiding with optical potentials will be limited fundamentally by diffraction [14], fundamental limitations to guiding condensates with microfabricated surfaces are not expected for an atom-surface separation in excess of $1 \mu \mathrm{m}$ [15].

In this Letter, we demonstrate that a Bose-Einstein condensate (BEC) transported with optical tweezers can be transferred into a microtrap on a substrate. Such condensates contained five times more atoms than those created in a similar microtrap [12]. We released the BEC from the microfabricated magnetic trap into a single-wire magnetic waveguide and studied its propagation. Condensates were observed to propagate $12 \mathrm{~mm}$ before exiting the field-of-view of our imaging system. The atoms propagated with a longitudinal kinetic energy in excess of the transverse confinement energy of the waveguide. This regime is important for atom interferometry because it reduces sensitivity to imperfections in the interferometer and the probability of atoms being reflected from interferometric elements.

We observed single-mode (excitation-less) BEC propagation along homogeneous segments of the waveguide.
Transverse excitations were created in condensates propagating through perturbations in the guiding potential. These perturbations resulted from geometric deformations of the current carrying wires on the substrate. Finer imperfections were observed when trapped condensates were brought closer to the surface of the microchip as evidenced by the longitudinal fragmentation of the cloud.

The apparatus used for this experiment was recently described in Ref. [11. Condensates containing over $10^{7}$ ${ }^{23} \mathrm{Na}$ atoms were created in the $\left|F, m_{F}\right\rangle=|1,-1\rangle$ state in a macroscopic Ioffe-Pritchard magnetic trap using forced radio frequency $(\mathrm{RF})$ evaporative cooling [16]. An optical tweezers beam then captured the BEC and transported it to within $500 \mu \mathrm{m}$ of the microfabricated structures. The microchip was contained in an auxiliary "science" chamber isolated from the BEC production chamber by bellows and a gate valve. The translation distance of the tweezers was $\approx 30 \mathrm{~cm}$ and was traversed in $1250 \mathrm{~ms}$. This quick transfer allowed for reliable delivery of condensates containing $2-3 \times 10^{6}$ atoms into the science chamber.

A schematic of the microchip onto which the BEC was loaded is shown in Fig. 1. The microfabricated wires lie on a $600 \mu \mathrm{m}$ thick silicon substrate. They are $50 \mu \mathrm{m}$ wide and electroplated with copper to a height of $10 \mu \mathrm{m}$. The minimum separation distance between wires is $50 \mu \mathrm{m}(100 \mu \mathrm{m}$ center-to-center $)$. The silicon wafer was mounted on an aluminium block for heat conduction purposes. Macroscopic leads that extended to a vacuum feedthrough were attached to the wafer using a gap welding technique.

The BEC was initially loaded into a Z-wire trap [7] formed by currents $I_{1}$ and $I_{2}$ along with an orthogonal magnetic bias field $B_{\perp}$. Typical loading conditions were $I_{1}=I_{2}=1200 \mathrm{~mA}$ and $B_{\perp}=5.4 \mathrm{G}$, corresponding to a separation of $450 \mu \mathrm{m}$ between the BEC and the microchip. A longitudinal bias field, $B_{\|}$, tailored the transverse trap frequency while nominally keeping the trap center in the same location relative to the surface of the microchip. Transfer efficiency from the optical tweezers to the Z-wire trap was near unity and BEC lifetimes over $10 \mathrm{~s}$ were observed with the application of a RF 


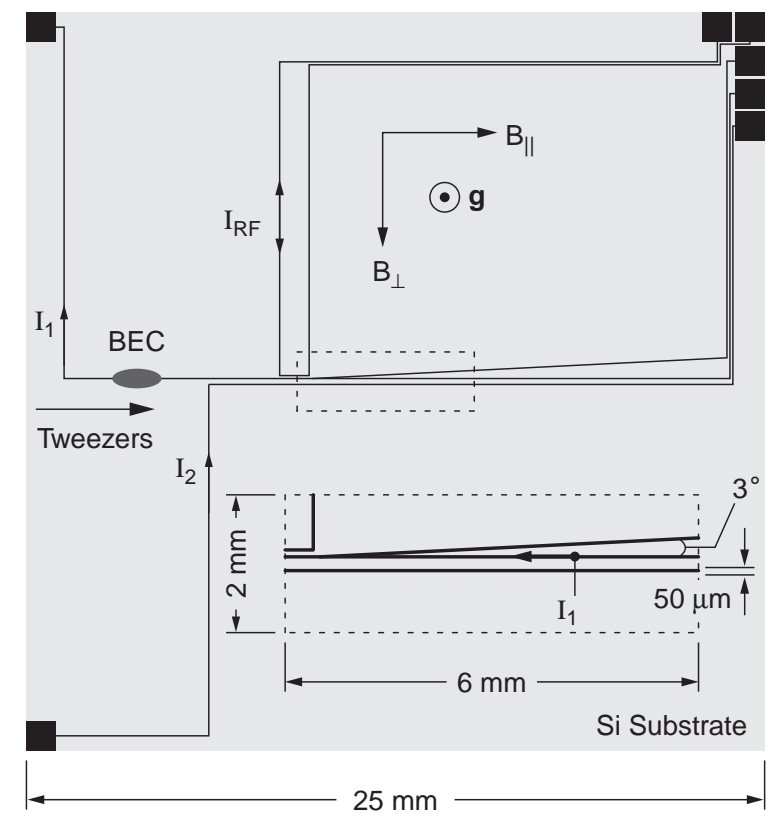

FIG. 1: Microfabricated magnetic trap and waveguide. Optical tweezers loaded a Bose-Einstein condensate into the Zwire trap formed by currents $I_{1}$ and $I_{2}$ in conjunction with the magnetic bias field $B_{\perp}$. Lowering $I_{2}$ to zero released the condensate into a single-wire magnetic waveguide. Atom flow was from left to right. The inset shows the widening of the waveguide wire in the region where another wire merges with it at a small angle. The only current flowing in the inset is $I_{1}$. The condensate was trapped above the plane of the paper and the gravitational force, $\mathbf{g}$, points out of the page. All microfabricated features are drawn to scale.

shield 16] produced by the current $I_{R F}$ on an auxiliary wire as shown in Fig. 1.

The BEC was transferred into the waveguide by linearly ramping the current $I_{2}$ to zero in $250 \mathrm{~ms}$. The atoms were smoothly accelerated into the waveguide by the remaining endcap of the Z-wire trap. Downstream, the effect of this endcap was negligible and we observed BEC propagation at a constant velocity of $3.0 \mathrm{~cm} / \mathrm{s}$ after a propagation distance of $4 \mathrm{~mm}$ for $I_{1}=1200 \mathrm{~mA}$. Upon releasing the BEC from the Z-wire trap, its longitudinal velocity was controlled by applying an external magnetic field gradient of variable amplitude for a fixed time. The field gradient was linearly ramped up and down over $6.5 \mathrm{~ms}$ to prevent creating excitations and was held constant for $52 \mathrm{~ms}$. With modest gradients of $0-0.6 \mathrm{G} / \mathrm{cm}$, we were able to vary the atomic velocity over the range $3.0-6.6 \mathrm{~cm} / \mathrm{s}$ corresponding to longitudinal kinetic energies in the range $25-120 \mathrm{kHz}$.

Releasing the BEC into the waveguide produced little variation in its transverse confinement and position relative to the microchip. The longitudinal potential experienced by the propagating atoms was determined by the local magnetic field (due to the Zeeman interaction) and vertical position of the guide center (due to the gravitational interaction). For ${ }^{23} \mathrm{Na}$ atoms in the $|1,-1\rangle$ state, the Zeeman energy is $700 \mathrm{~Hz} / \mathrm{mG}$ and the gravitational energy is $560 \mathrm{~Hz} / \mu \mathrm{m}$.

Perturbations to the guiding potential arise from geometric deformations of the current carrying wires on the substrate. The extent to which such deformations alter the potential experienced by the atoms depends on the atom-wire separation distance, $r$, longitudinal extent of the perturbation, $\ell$, wire width, $w$, and wire height, $h$. Under our guiding conditions $(r \gg w, h)$ the waveguide potential only responds to changes in the centroid of the current density. In general, only wire deformations with $\ell \gtrsim r$ will significantly perturb the guiding potential due to solid angle considerations.

Such a deformation $(\ell \approx 1 \mathrm{~mm}, r=450 \mu \mathrm{m})$ is depicted in the inset to Fig. 11. As the wire width varies during the bifurcation process, the centroid of the current density will shift in the plane of the microchip. This shift will be mirrored by a shift in the trajectory of the guide in a plane parallel to that of the microchip [17. Such a shift causes the guide axis to make an angle, $\theta$, with respect to its nominal trajectory along which $B_{\|}$and $B_{\perp}$ are aligned parallel and perpendicular respectively. As a result, the effective parallel, $B_{\|}^{\prime}$, and perpendicular, $B_{\perp}^{\prime}$, magnetic fields are found by the rotation

$$
\begin{aligned}
B_{\|}^{\prime} & =B_{\|} \cos \theta-B_{\perp} \sin \theta, \\
B_{\perp}^{\prime} & =B_{\perp} \cos \theta+B_{\|} \sin \theta,
\end{aligned}
$$

where $-\pi / 2 \leq \theta \leq \pi / 2$. $\theta$ is taken to be a positive(negative) angle for the specific case of atoms entering(exiting) the waveguide perturbation depicted in the inset to Fig. 1. 1 .

Changes in the effective parallel and perpendicular magnetic fields result in variations in the longitudinal potential experienced by the atoms due to the Zeeman and gravitational interactions respectively. Variations in the effective parallel magnetic field are given by (for small angles)

$$
\Delta B_{||}^{\prime}=-B_{\perp} \sin \theta .
$$

Thus, atoms entering(exiting) the perturbed guiding region will encounter a magnetic potential well(barrier). Furthermore, changes in the effective perpendicular bias field cause the atom-substrate distance to vary by (for small angles)

$$
\Delta r=-r \frac{\Delta B_{\perp}}{B_{\perp}}=-r \frac{B_{\|}}{B_{\perp}} \sin \theta .
$$

Thus, the guide center entering(exiting) the perturbed guiding region will shift towards(away from) the surface of the microchip and atoms will encounter a gravitational potential barrier(well). For the waveguide parameters used in this work, perturbations to the guiding potential 


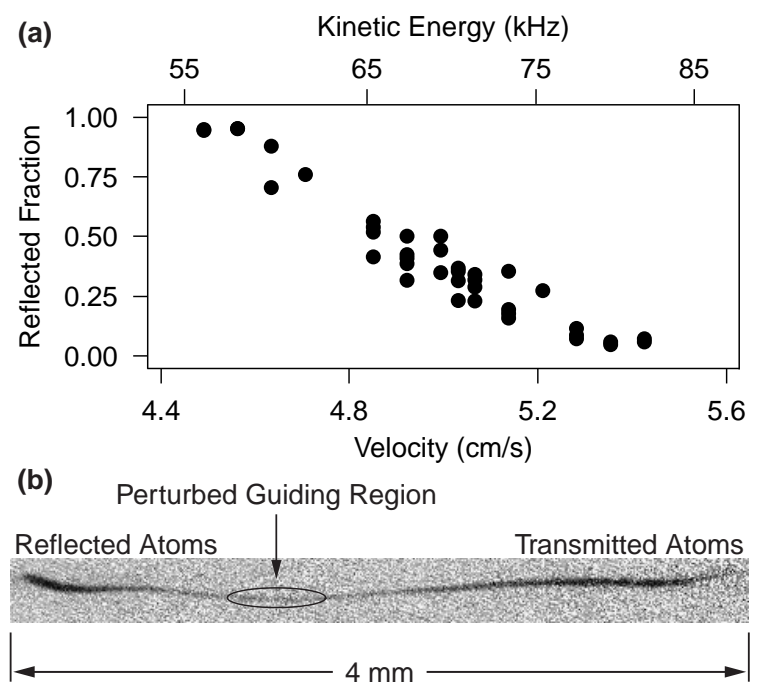

FIG. 2: Bose-Einstein condensates scattering from the waveguide perturbation shown in the inset to Fig. 1. (a) The fraction of atoms reflected as a function of incident velocity. (b) Absorption image after $8 \mathrm{~ms}$ of ballistic expansion of a partially transmitted condensate. The incident longitudinal velocity and kinetic energy were $5.0 \mathrm{~cm} / \mathrm{s}$ and $70 \mathrm{kHz}$ respectively. Waveguide parameters for all data were $I_{1}=1200 \mathrm{~mA}$, $B_{\perp}=5.4 \mathrm{G}, B_{\|}=3.6 \mathrm{G}$, and $\omega_{\perp}=2 \pi \times 84.5 \mathrm{~Hz}$.

are dominated by longitudinal variations in the Zeeman energy.

The effects of the perturbation depicted in the inset to Fig. 11 on BEC propagation were studied by varying the incident velocity of the atoms sent into the region. The BEC entered the perturbed guiding region after $\geq 100 \mathrm{~ms}$ of propagation, well after the external gradient used to accelerate the atoms was turned off. Condensates travelling below $4.5 \mathrm{~cm} / \mathrm{s}$ ( $55 \mathrm{kHz}$ kinetic energy) were totally reflected from the perturbation, while clouds at speeds above $5.4 \mathrm{~cm} / \mathrm{s}$ ( $80 \mathrm{kHz}$ kinetic energy) were entirely transmitted. Fig. 2 shows the results for intermediate atomic velocities.

The local magnetic field in the perturbed guiding region was measured by driving RF spin-flip transitions that removed atoms from the guide [16]. It was found that upon entering(exiting) the perturbed region the magnetic bottom of the guiding potential decreased(increased) by $50 \pm 10 \mathrm{kHz}$. The signs of these shifts are consistent with those predicted by Eq. 3. The magnitude of the shift in the Zeeman energy upon exiting the guide is also consistent with the onset of transmission through the perturbed guiding region as shown in Fig. 2(a). From Eq. 3 with $B_{\perp}=5.4$ G, the maximum angular deviation, $\theta_{m}$, of the waveguide trajectory necessary to produce a $50 \mathrm{kHz}$ perturbation to the Zeeman energy is $\theta_{m}=13 \mathrm{mrad}$. The corresponding vertical displacement of the guide center from Eq. A with $B_{\perp}=5.4 \mathrm{G}, B_{\|}=3.6 \mathrm{G}$, and $\theta_{m}=13 \mathrm{mrad}$ is $4 \mu \mathrm{m}$.
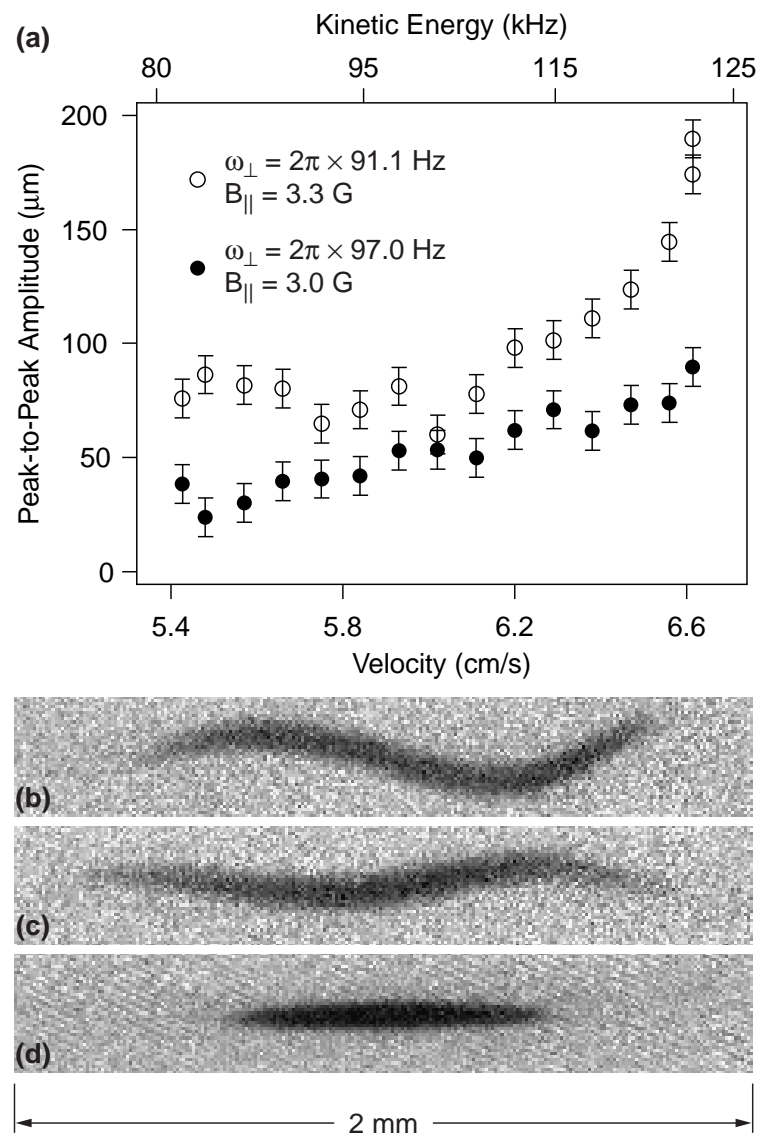

FIG. 3: Velocity dependence of Bose-Einstein condensate excitations after propagating through the waveguide perturbation depicted in the inset to Fig. 1. (a) Peak-to-peak amplitudes of condensate excitations after $15 \mathrm{~ms}$ of ballistic expansion versus velocity, $v$. Absorption images of condensates with $v=6.5 \mathrm{~cm} / \mathrm{s}$ after $15 \mathrm{~ms}$ of ballistic expansion for (b) $\omega_{\perp}=2 \pi \times 91.1 \mathrm{~Hz}$ and $B_{\|}=3.3 \mathrm{G}$ and $(\mathrm{c}) \omega_{\perp}=2 \pi \times 97.0 \mathrm{~Hz}$ and $B_{\|}=3.0 \mathrm{G}$. (d) Typical condensate after $10 \mathrm{~ms}$ of ballistic expansion $\left(N \approx 2 \times 10^{6}\right.$ atoms, $v=6.3 \mathrm{~cm} / \mathrm{s}$, $\omega_{\perp}=2 \pi \times 84.5 \mathrm{~Hz}$, and $B_{\|}=3.6 \mathrm{G}$ ) just prior to entering the perturbed guiding region. The wire current, $I_{1}=1200 \mathrm{~mA}$, and perpendicular bias field, $B_{\perp}=5.4 \mathrm{G}$, were held fixed for all data. All transmitted condensates propagated $4 \mathrm{~mm}$ beyond the perturbed guiding region before being imaged.

This yields a gravitational potential variation of $2.2 \mathrm{kHz}$, which is small compared to the $50 \mathrm{kHz}$ Zeeman energy shifts associated with the perturbation.

In the propagation regime where the longitudinal kinetic energy of the BEC is large compared to the transverse confinement energy of the guide, perturbations involving transverse shifts in the guide trajectory are expected to transversely excite incident condensates. Fig. 3 depicts such transverse excitations for condensates transmitted through the waveguide perturbation depicted in the inset to Fig. 11. The incident BEC velocity was in the range $5.4-6.6 \mathrm{~cm} / \mathrm{s}$ corresponding to longitudinal kinetic energies in the range $80-120 \mathrm{kHz}$. Excitations 
were characterized by the peak-to-peak amplitude of the transverse displacement of the BEC. The imaging axis only provided sensitivity to transverse excitations in the plane normal to the surface of the microchip.

Fig. 3(a) shows a clear increase in the excitation amplitude with increasing velocity. Furthermore, the excitation amplitude for fixed incident atomic velocity should increase when either the ratio of longitudinal kinetic energy to transverse confinement energy or the size of the perturbation increases. By increasing $B_{\|}$we simultaneously lowered the trap frequency and, according to Eq. 4, increased the vertical displacement of the guide center. This resulted in an observed increase in excitation amplitude for all velocities studied.

The nearly sinusoidal nature of the BEC excitations shown in Fig. 3(b) and (c) indicates that we were primarily exciting the dipole mode of the BEC. Further evidence for this comes from the fact that we observed little variation in the excitation amplitude as a function of the propagation time after exiting the perturbed guiding region. Conversely, transmitted condensates showed strong signs of higher order excitations when $B_{||}$was increased beyond $3.6 \mathrm{G}$ corresponding to $\omega_{\perp} \leq 2 \pi \times 84.5 \mathrm{~Hz}$. The absorption images deviated visibly from a smooth sinusoidal shape. In addition, the measured excitation amplitude depended strongly on propagation time indicting the phasing and dephasing of several excitation modes.

Because the perturbations to the magnetic potential above a single wire are due to its geometric deformations, one expects such perturbations to increase as the trap center is brought closer to the surface of the microchip. We observed longitudinal fragmentation of the BEC as the atoms were brought to within $150 \mu \mathrm{m}$ of the surface (Fig. 4). At $55 \mu \mathrm{m}$ from the surface the potential developed axial variations with a characteristic length scale of $100-150 \mu \mathrm{m}$. These variations could be magnetic or gravitational in origin.

In conclusion, we used optical tweezers to load a microfabricated magnetic trap with $2-3 \times 10^{6}$ condensate atoms and subsequently released the atoms into a magnetic waveguide. Our setup merges the scientific potential of microfabricated structures with the proven robustness of conventional BEC production techniques. In this work, the linear density of atoms propagating along the waveguide was $10-20$ times larger than the one-dimensional criterion, which for ${ }^{23} \mathrm{Na}$ is $n_{1 D} \approx 100 \mu \mathrm{m}^{-1}[18$. Due to their lack of longitudinal confinement, waveguides are well suited for creating highly elongated clouds making them ideal for minimizing mean field effects [19] in condensate interferometers and for studies of one-dimensional physics including phase fluctuations [20, 21, 22 and gases of impenetrable bosons 21, 23].

This work was funded by ONR, NSF, ARO, NASA, and the David and Lucile Packard Foundation. A.E.L. acknowledges additional support from NSF. We are in-

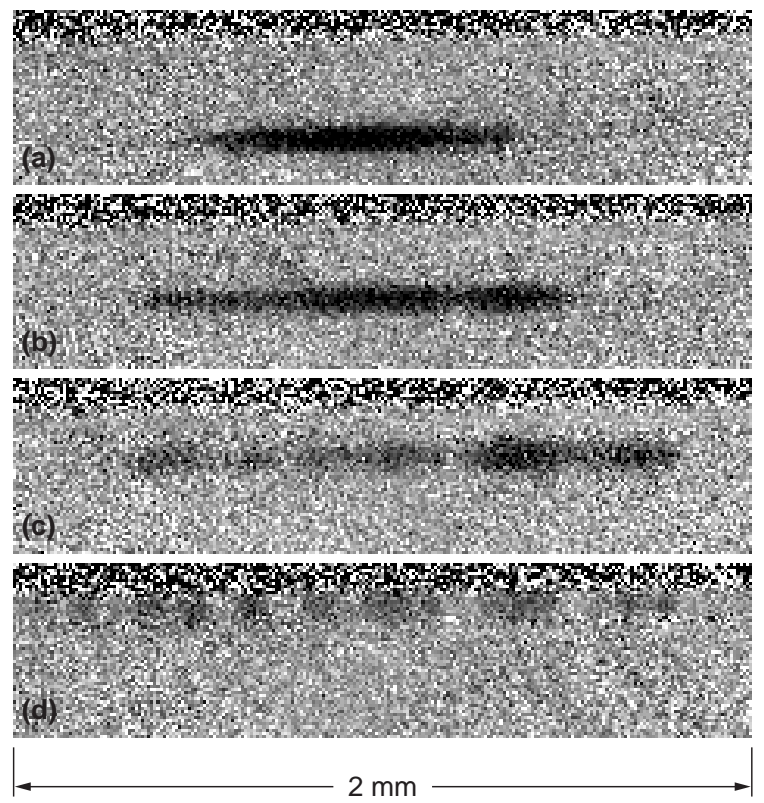

FIG. 4: Fragmented structure of Bose-Einstein condensates brought close to the substrate. Absorption images after $5 \mathrm{~ms}$ of ballistic expansion from the Z-wire trap shown in Fig. 1. The atom-substrate separation was (a) $190 \mu \mathrm{m}$, (b) $145 \mu \mathrm{m}$, (c) $100 \mu \mathrm{m}$, and (d) $55 \mu \mathrm{m}$. For all images, the condensate started in a trap with $I_{1}=I_{2}=540 \mathrm{~mA}, B_{\perp}=5.4 \mathrm{G}$, and $B_{\|}=0.3 \mathrm{G}$ corresponding to an atom-substrate separation of $200 \mu \mathrm{m}$. The condensate was translated towards the substrate by lowering the wire currents linearly over $500 \mathrm{~ms}$. The atoms were held at their final position for $100 \mathrm{~ms}$ prior to trap shut off.

debted to S. Gupta for contributions to the apparatus, E. Tsikata for experimental assistance, the MIT Microsystems Technology Laboratories for fabricating the microchip used in this work, M. Prentiss and M. Vengalattore for valuable discussions, and A.D. Cronin and D. Schneble for critical readings of the manuscript.

* URL: http://cua.mit.edu/ketterle_group/

[1] M. Inguscio, S. Stringari, and C. E. Wieman, eds., BoseEinstein Condensation in Atomic Gases, Proceedings of the International School of Physics Enrico Fermi, Course CXL (IOS Press, Amsterdam, 1999).

[2] J. D. Weinstein and K. G. Libbrecht, Phys. Rev. A 52, 4004 (1995).

[3] J. H. Thywissen, M. Olshanii, G. Zabow, M. Drndić, K. S. Johnson, R. M. Westervelt, and M. Prentiss, Eur. Phys. J. D 7, 361 (1999).

[4] J. Fortagh, A. Grossmann, C. Zimmermann, and T. W. Hänsch, Phys. Rev. Lett. 81, 5310 (1998).

[5] J. Denschlag, D. Cassettari, and J. Schmiedmayer, Phys. Rev. Lett. 82, 2014 (1999).

[6] M. Key, I. G. Hughes, W. Rooijakkers, B. E. Sauer, E. A. Hinds, D. J. Richardson, and P. G. Kazansky, Phys. Rev. 
Lett. 84, 1371 (2000).

[7] J. Reichel, W. Hänsel, and T. W. Hänsch, Phys. Rev. Lett. 83, 3398 (1999).

[8] D. Müller, D. Z. Anderson, R. J. Grow, P. D. D. Schwindt, and E. A. Cornell, Phys. Rev. Lett. 83, 5194 (1999).

[9] N. H. Dekker, C. S. Lee, V. Lorent, J. H. Thywissen, S. P. Smith, M. Drndić, R. M. Westervelt, and M. Prentiss, Phys. Rev. Lett. 84, 1124 (2000).

[10] R. Folman, P. Krüger, D. Cassettari, B. Hessmo, T. Maier, and J. Schmiedmayer, Phys. Rev. Lett. 84, 4749 (2000).

[11] T. L. Gustavson, A. P. Chikkatur, A. E. Leanhardt, A. Görlitz, S. Gupta, D. E. Pritchard, and W. Ketterle, Phys. Rev. Lett. 88, 020401 (2002).

[12] H. Ott, J. Fortagh, G. Schlotterbeck, A. Grossmann, and C. Zimmermann, Phys. Rev. Lett. 87, 230401 (2001).

[13] W. Hänsel, P. Hommelhoff, T. W. Hänsch, and J. Reichel, Nature 413, 498 (2001).

[14] K. Bongs, S. Burger, S. Dettmer, D. Hellweg, J. Arlt, W. Ertmer, and K. Sengstock, Phys. Rev. A. 63, 031602(R) (2001).

[15] C. Henkel and W. Wilkens, Europhys. Lett. 47, 414
(1999).

[16] W. Ketterle, D. S. Durfee, and D. M. Stamper-Kurn, in [1], pp. 67-176.

[17] Analogously, the trajectory will vary in a plane normal to the microchip for variations in the wire height.

[18] A. Görlitz, J. M. Vogels, A. E. Leanhardt, C. Raman, T. L. Gustavson, J. R. Abo-Shaeer, A. P. Chikkatur, S. Gupta, S. Inouye, T. Rosenband, et al., Phys. Rev. Lett. 87, 130402 (2001).

[19] A. Smerzi, S. Fantoni, S. Giovanazzi, and S. R. Shenoy, Phys. Rev. Lett. 79, 4950 (1997).

[20] S. Dettmer, D. Hellweg, P. Ryytty, J. J. Arlt, W. Ertmer, K. Sengstock, D. S. Petrov, G. V. Shlyapnikov, H. Kreutzmann, L. Santos, et al., Phys. Rev. Lett. 87, 160406 (2001).

[21] D. S. Petrov, G. V. Shlyapnikov, and J. T. M. Walraven, Phys. Rev. Lett. 85, 3745 (2000).

[22] J. O. Andersen, U. Al Khawaja, and H. T. C. Stoof, Phys. Rev. Lett. 88, 070407 (2002).

[23] V. Dunjko, V. Lorent, and M. Olshanii, Phys. Rev. Lett. 86, 5413 (2001). 